\title{
The Association between FLAIR Vascular Hyperintensity and Stroke Outcome Varies with Time from Onset
}

\author{
(D)W.J. Shang, (DH.B. Chen, (D) L.M. Shu, DH.Q. Liao, (D) X.Y. Huang, (D) S. Xiao, and DH. Hong
}

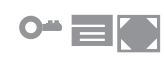

\begin{abstract}
BACKGROUND AND PURPOSE: FLAIR vascular hyperintensity has been recognized as a marker of collaterals in ischemic stroke, but the impact on outcome is still controversial. We hypothesized that the association between FLAIR vascular hyperintensity and outcome varies with time.
\end{abstract}

MATERIALS AND METHODS: We included 459 consecutive patients with middle cerebral artery stroke and divided them into 3 groups by symptom-to-MR imaging time (group 1, $\leq 7$ days; group 2, 8-14 days; group 3, $\geq 15$ days). The FLAIR vascular hyperintensity score, ranging from 0 to 3 points, was based on territory distributions of different MCA segments. The associations between FLAIR vascular hyperintensity and outcome with time were analyzed qualitatively and quantitatively.

RESULTS: No patients underwent MR imaging within 6 hours of onset. The proportion of FLAIR vascular hyperintensity $(+)$ and severe stenosis or occlusion of MCA was not significantly dependent on time. In groups 1 and 2, FLAIR vascular hyperintensity $(+)$ was significantly associated with larger lesions, the prevalence of flow injury, and unfavorable outcome ( $m R S \geq 2)$. There were no such associations in group 3. Multiple logistic regressions demonstrated that FLAIR vascular hyperintensity $(+)$ was an independent risk factor for unfavorable outcome in group 2. Infarction volume tended to increase with the increase of the distal FLAIR vascular hyperintensity score in groups 1 and 2, while declining in group 3.

CONCLUSIONS: FLAIR vascular hyperintensity is associated with unfavorable outcome within 6 hours to 14 days of onset, while the wider distribution of distal FLAIR vascular hyperintensity may be favorable beyond 14 days of onset in MCA infarction. Symptom-to-MR imaging time should be considered when assessing the prognostic value of FLAIR vascular hyperintensity.

ABBREVIATION: FVH = FLAIR vascular hyperintensity

erebral ischemic damage depends on both the degree and duration of hypoperfusion. However, collaterals neither de-

\section{Received March 13, 2019; accepted after revision June 17}

From the Department of Neurology (W.J.S., H.B.C., L.M.S., S.X., H.H.), National Key Clinical Department and Key Discipline of Neurology, The First Affiliated Hospital, Sun Yat-sen University, Guangzhou, China; and Department of Neurology (L.M.S., H.Q.L., X.Y.H.), The Seventh Affiliated Hospital, Sun Yat-sen University, Shenzhen, China.

W.J. Shang and H.B. Chen contributed to the work equally.

The study was supported by grants from: National Natural Science Foundation of China (No. 81671134); Medical Scientific Research Foundation of Guangdong Province of China (No. A2017219); Guangzhou Science and Technology Programme (No. 2014Y2-00502); Guangdong Provincial Key Laboratory for Diagnosis and Treatment of Major Neurological Diseases (No. 2017B030314103); Southern China International Cooperation Base for Early Intervention and Functional Rehabilitation of Neurological Diseases (No. 2015B050501003); Guangdong Provincial Engineering Center for Major Neurological Disease Treatment; and Guangdong Provincial Translational Medicine Innovation Platform for Diagnosis and Treatment of Major Neurological Disease.

Please address correspondence to Hua Hong, Department of Neurology, National Key Clinical Department and Key Discipline of Neurology, The First Affiliated Hospital, Sun Yat-sen University, 58 Zhongshan 2nd Rd, Guangzhou, Guangdong Province, China, 510080; e-mail: hhsums@126.com velop rapidly nor remain invariant after arterial occlusion. Some collaterals such as leptomeningeal anastomoses may be anatomically present, though enhanced capacity for cerebral blood flow likely requires time to develop. ${ }^{1-5}$ Angiogenesis induced by hypoxia and increased fluid shear stress also requires several days to weeks. ${ }^{1,2}$ Furthermore, the incipient development of collaterals does not guarantee their persistence. ${ }^{1}$ Therefore, the prognostic significance of an observation of the presence of collaterals after ischemic stroke varies with time.

FLAIR vascular hyperintensity (FVH), seen in some patients with ischemic stroke, is known to be associated with stenosis and occlusion of vessels and the attendant slow flow. ${ }^{6-9} \mathrm{FVH}$ is also thought to be a marker of collaterals and a predictor of outcome. ${ }^{4,6-23}$ However, the relationship between these interpreta-

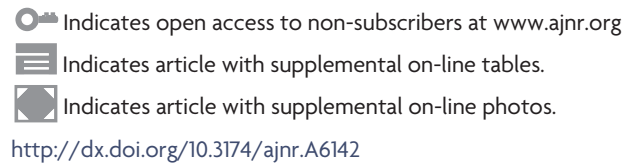


tions is confusing. FVH may represent slow collateral flow effective in maintaining perfusion to penumbral regions, restricting the progress of ischemic lesions and improving outcome. ${ }^{10-17}$ However, FVH can also seem to correspond to a perfusion deficit, larger lesions, and poor outcomes. ${ }^{6-8,18-23}$ The exact reason for such a huge discrepancy is still unclear. In addition to the differences in patient populations and FVH classifications, we found that many reported symptom-to-MR imaging times ranged from 3 hours to several days. Additionally, Maeda et $\mathrm{al}^{24}$ showed that FVH decreased with time, indicating that differences in symptom-to-MR imaging time might account for the discrepancy.

Moreover, most studies ${ }^{6-8,10-18,21,22}$ were performed during the superacute or acute stages of ischemic stroke; however, many patients with subacute or even chronic ischemic strokes present to hospitals and undergo MR imaging. FVH has been observed in areas supplied by collateral blood flow in patients with symptomto-MR imaging times that are several weeks in duration. ${ }^{25}$ Given that FVH is easily obtained via MR imaging, it could serve as a helpful predictive measure in patients with ischemic stroke.

We hypothesized that the prognostic value of FVH in ischemic stroke might change with the MR imaging observation time relative to onset. Therefore, we here evaluate the dependence of the relationship between FVH and outcome on symptom-to-MR imaging time, in cases of middle cerebral artery ischemic stroke.

\section{MATERIALS AND METHODS Study Population}

We retrospectively analyzed the records of patients with ischemic stroke treated in the First Affiliated Hospital of Sun Yat-sen University from April 2009 to February 2017. The inclusion criteria were the following: 1) patients with responsible lesions within the MCA distribution territory; 2) patients with FLAIR and 3D timeof-flight MRA images; 3) patients with ipsilateral M1-MCA stenosis or occlusion identified by 3D time-of-flight MRA; and 4) patients with a complete evaluation, which included a personal history, vascular risk factors (smoking, hypertension, diabetes mellitus, hyperlipidemia, previous stroke/transient ischemic attack, coronary artery disease, and atrial fibrillation), routine blood tests, and cardiologic work-up. Scores on the NIHSS at admission, $\mathrm{mRS}$ at discharge, and hospitalization days were also collected. We excluded the following; 1) patients with concomitant anterior cerebral artery or posterior circulation strokes; 2) patients with severe artifacts on FLAIR images; 3) patients with severe stenosis or occlusion of the ipsilateral internal carotid artery; and 4) patients having undergone endovascular therapy. Patients were divided into 3 groups according to symptom-to-MR imaging time: group $1, \leq 7$ days; group 2, 8-14 days; group 3, $\geq 15$ days.

\section{MR Imaging}

MR imaging was performed on 3T scanners (Magnetom Trio and Magnetom Verio; Siemens, Erlangen, Germany) with a 12-channel head coil. The neuroimaging protocol comprised T1WI (TR, $500 \mathrm{~ms}$; TE, $8.9 \mathrm{~ms}$ ), T2WI (TR, $4000 \mathrm{~ms}$; TE, $100 \mathrm{~ms}$ ), FLAIR (TR, $9000 \mathrm{~ms}$; TE, $111 \mathrm{~ms}$; TI, $2500 \mathrm{~ms}$ ), and DWI (TR, $5800 \mathrm{~ms}$; TE, $100 \mathrm{~ms}$; matrix number, $384 \times 384$; two b-values of 0 and $1000 \mathrm{~s} / \mathrm{mm}^{2}$ ). All patients' imaging data were independently reviewed by 2 readers who were blinded to the clinical data and follow-up. Reader A had 18 years of neuroradiology experience, and reader B had 6 years of neuroradiology experience.

\section{FVH}

FVHs were defined as focal, tubular, or serpentine hyperintensities in the subarachnoid space relative to CSF and corresponding to the typical arterial course. ${ }^{11}$ On the basis of the location and extent, FVHs were classified into proximal and distal FVHs. Proximal FVHs were defined as the presence of FVHs only within the territories of the MCA M1 and/or M2 segments. Distal FVHs were defined as the presence of FVHs in the MCA M3 and/or distal segments, which were further classified into anterior and posterior for the superior and inferior trunks of the MCA, respectively. The M3-segment FVHs, anterior FVHs, and posterior FVHs were all scored as 1 point.

\section{Lesion Quantification}

We used the ASPECTS on MR imaging. ${ }^{26}$ The vascular territories were classified into perforator, pial, border zone, and large-territory infarct. ${ }^{27}$

\section{Angiography}

The signal intensity of the MCA on the intracranial MRA was visually classified into the following 4 grades according to ability to visualize the MCA: All M3 branches of the MCA were visualizable to the cortical surface (grade A); $\geq 1 \mathrm{M} 3$ branch could not be visualized on the cortical surface (grade B); the superior or inferior trunk of the MCA or $\geq 1$ M2 branch could not be visualized along its course (grade C); and the M1 could not be visualized along its course (grade D) (On-line Fig 1). ${ }^{28}$ Posterior cerebral artery laterality was considered to be present if $\geq 1$ segmental extent of the ipsilateral posterior cerebral artery was observable on MRA. ${ }^{5}$

\section{Statistical Analysis}

The $\kappa$ coefficient was used to assess interobserver agreement for FVH. Continuous variables with a normal distribution were described as mean $\pm \mathrm{SD}$, and non-normally distributed variables were described as median and interquartile range. We compared variables between the patients with and without $\mathrm{FVH}$ in each group using a Mann-Whitney $U$ test, Student $t$ test, Pearson $\chi^{2}$ test, or $\chi^{2}$ test with continuity correction depending on the type of variable. We further compared FVH scores and baseline characteristics in all 3 groups using the Kruskal-Wallis-test or Pearson $\chi^{2}$ test as appropriate. A multiple binary logistic regression analysis was applied to identify independent predictors of unfavorable scores on the modified Rankin Scale at discharge ( $\mathrm{mRS} \geq 2$ ), signifying an unfavorable outcome. All covariates with a $P$ value $\leq .1$ in a univariate analysis were entered into this logistic regression model. The results are presented as odds ratios and the 95\% confidence intervals. A 2-tailed value of $P<.05$ indicated statistical significance (SPSS for Windows, Version 20.0; IBM, Armonk, New York).

\section{RESULTS}

In total, 579 consecutive patients met all the inclusion criteria, and 120 patients were excluded (concomitant anterior cerebral 
Table 1: Clinical and demographic patient characteristics by symptom-to-MRI time and the presence of FVH

\begin{tabular}{|c|c|c|c|c|c|c|c|c|c|}
\hline \multirow[b]{2}{*}{ Characteristics } & \multicolumn{3}{|c|}{ Group $1(n=191)$} & \multicolumn{3}{|c|}{ Group $2(n=164)$} & \multicolumn{3}{|c|}{ Group $3(n=104)$} \\
\hline & $\begin{array}{l}\text { FVH (-) } \\
(n=74)\end{array}$ & $\begin{array}{l}\text { FVH (+) } \\
(n=117)\end{array}$ & $P$ Value & $\begin{array}{l}\text { FVH (-) } \\
(n=63)\end{array}$ & $\begin{array}{l}\text { FVH (+) } \\
(n=101)\end{array}$ & $P$ Value & $\begin{array}{l}\text { FVH (-) } \\
(n=44)\end{array}$ & $\begin{array}{l}\text { FVH (+) } \\
(n=60)\end{array}$ & $P$ Value \\
\hline Women & $27(36.5)$ & $37(31.6)$ & .53 & $20(31.7)$ & $27(26.7)$ & .60 & $10(22.7)$ & $20(33.3)$ & .28 \\
\hline Age $(y r)$ & $60.7 \pm 14.3$ & $63.9 \pm 12.5$ & .11 & $66(46-73)$ & $65.5(54-74)$ & .88 & $55.1 \pm 13.7$ & $63.6 \pm 11.0$ & $.001^{\mathrm{b}}$ \\
\hline Symptom-to-admission time (days) & $3(2-4)$ & $2(1-4)$ & .05 & $5(4-7)$ & $4(3-7)$ & .21 & $16.5(12-31)$ & $19.5(12-30.75)$ & .79 \\
\hline \multicolumn{10}{|l|}{ Risk factors } \\
\hline Hypertension & $58(78.4)$ & $82(70.1)$ & .24 & $35(55.6)$ & $70(69.3)$ & .09 & $31(70.5)$ & $45(75)$ & .66 \\
\hline Diabetes mellitus & $29(39.2)$ & $28(23.9)$ & $.03^{\mathrm{b}}$ & $16(25.4)$ & $36(35.6)$ & .23 & $12(27.3)$ & $13(21.7)$ & .64 \\
\hline Hypercholesterolemia & $24(32.4)$ & $43(36.8)$ & .64 & $24(38.1)$ & $30(29.7)$ & .31 & $8(18.2)$ & $8(13.3)$ & .59 \\
\hline Smoker & $24(32.4)$ & $52(44.4)$ & .13 & $25(39.7)$ & $48(47.5)$ & .34 & 19 (43.2) & $26(43.3)$ & 1.00 \\
\hline Coronary heart disease & $5(6.8)$ & $6(5.1)$ & .88 & $1(1.6)$ & $6(5.9)$ & .35 & $6(13.6)$ & $5(8.3)$ & .59 \\
\hline Atrial fibrillation & $4(5.4)$ & $8(6.8)$ & .93 & $2(3.2)$ & $1(1.0)$ & .88 & $1(2.3)$ & $2(3.3)$ & 1.00 \\
\hline Previous stroke & $18(24.3)$ & $29(24.8)$ & 1.00 & $9(14.3)$ & $25(24.8)$ & .12 & $9(20.5)$ & $19(31.7)$ & .27 \\
\hline NIHSS score at admission & $4(2-7)$ & $6(3-10)$ & $.02^{\mathrm{b}}$ & $4(2-9)$ & $5(2-9)$ & .32 & $5(2-10)$ & $4.5(2-9)$ & .56 \\
\hline Hospitalization (days) & $10.5(8-13)$ & $13(10-16)$ & $.001^{\mathrm{b}}$ & $12(9-15)$ & $13(9-15)$ & .95 & $13.5(8-15)$ & $12.5(9.25-16)$ & .94 \\
\hline \multicolumn{10}{|l|}{ Outcome at discharge } \\
\hline mRS & $2(1-3)$ & $3(2-4)$ & $<.001^{\mathrm{b}}$ & $2(1-3)$ & $3(2-4)$ & .10 & $2.5(1-4)$ & $3(1.25-4)$ & .54 \\
\hline $\mathrm{mRS} \geq 2$ & $54(73.0)$ & $100(85.5)$ & $.04^{\mathrm{b}}$ & $43(68.3)$ & $85(84.2)$ & $.02^{\mathrm{b}}$ & $31(70.5)$ & $45(75.0)$ & .66 \\
\hline $\mathrm{mRS} \geq 3$ & $31(41.9)$ & $70(59.8)$ & $.02^{\mathrm{b}}$ & $29(46.0)$ & $52(51.5)$ & .52 & $22(50.0)$ & $34(56.7)$ & .55 \\
\hline
\end{tabular}

Note:-(-) indicates negative; (+), positive.

${ }^{a}$ Data are No. (\%), mean \pm SD, and median (interquartile range)

${ }^{\mathrm{b}} \mathrm{P}<.05$.

artery or posterior circulation strokes, $n=27$; severe artifacts on FLAIR, $n=18$; severe stenosis or occlusion of the ipsilateral internal carotid artery, $n=47$; endovascular therapy, $n=28)$. We finally included 459 patients, and FVH was observed in 278 patients $(60.6 \%)$. The interobserver agreement for FVH was $\kappa=$ 0.88 (95\% CI, 0.68-1.00). The proportion of observed FVH $(+)$ was not significantly dependent on time $(61.3 \%, 61.6 \%$, and $57.7 \% ; P=.79)$. Details of the clinical and demographic characteristics of patients by symptom-to-MR imaging time and the presence of FVH are presented in Table 1. In group 1, patients with FVH demonstrated a higher NIHSS score at admission $(P=$ $.02)$, a longer hospitalization $(P=.001)$, a higher $\mathrm{mRS}$ at discharge $(P=.001)$, and a higher prevalence of unfavorable outcome (mRS $\geq 2 ; P=.04$ ) or poor outcome (mRS $\geq 3 ; P=.02$ ) than those without FVH. In group 2, patients with FVH demonstrated a higher prevalence of unfavorable outcome ( $\mathrm{mRS} \geq 2$; $P=.02$ ). However, in group 3 , we found no significant difference in outcome between patients with and without FVH.

Details of the radiologic characteristics of patients by symptom-to-MR imaging time and FVH status are presented in Online Table 1, Figs 1-3, and On-line Figs 2-4. No patients underwent MR imaging within 6 hours of onset. More patients with FVH demonstrated severe stenosis or occlusion of the MCA than those without it, independent of time (group 1, $P<.001$; group 2 , $P<.001$; group $3, P=.001$ ). In groups 1 and 2, patients with $\mathrm{FVH}$ demonstrated larger cortical extents of MCA and total lesion scores than those without it (group $1, P<.001, P<.001$; group 2, $P=.003, P=.003$ ), and poorer MCA signal intensities (grades B-D; group $1, P<.001$; group $2, P<.001)$. However, these differences were absent in group $3(P=.73, P=.45, P=.18)$. We observed no significant differences in FVH scores $(P=0.25)$ across the 3 groups. The MCA cortical area and total lesion volume tended to increase with the distal FVH score in groups 1 and 2 and decrease in group 3 (Fig 3). Details of the baseline characteristics of the 3 groups are presented in On-line Table 2. There were no differences among the 3 groups, apart from hypercholesterolemia.
Variables associated with an unfavorable clinical outcome are shown in On-line Table 3. In group $1, \operatorname{sex}(P=.01)$, NIHSS score on admission $(P<.001)$, infarct volume $(P=.001)$, and FVH $(P=.04)$ were included in our analysis. After we adjusted for representative variables, $\mathrm{FVH}(+)$ did not appear as an independent predictor of unfavorable outcome $(P=.14)$. In group 2, age $(P=.003)$, NIHSS score on admission $(P<.001)$, infarct volume $(P=.001)$, FVH $(P=.002)$, posterior cerebral artery laterality $(P=.008)$, and MCA severe stenosis or occlusion $(P=$ $.006)$ were included in our analysis. After we adjusted for representative variables, $\mathrm{FVH}(+)$ appeared as an independent predictor of unfavorable outcome (odds ratio, 2.95; 95\% CI, 1.06-8.18; $P=.04$ ). In group $3, \mathrm{FVH}(+)$ was not included in our analysis $(P=.66)$.

\section{DISCUSSION}

Our novel results demonstrate that the clinical value of FVH varies with time. Within 6 hours to 14 days of onset, $\mathrm{FVH}(+)$ is associated with greater lesion volume, poorer MCA signal intensity, and unfavorable outcome. These associations disappear beyond 14 days of onset. In addition, the lesion volume tended to increase with the distal FVH score within 6 hours to 14 days of onset and to decrease beyond 14 days of onset. Therefore, the symptom-to-MR imaging time, not just the presence or distribution of FVH, played an important role in predicting stroke outcomes in this study.

FVH is recognized as a marker of slow flow induced by severe stenosis or occlusion of vessels. ${ }^{6-9}$ The presence of FVH ranges from $44.1 \%$ to $100 \%$ in patients with stroke within 24 hours of onset, ${ }^{11,29}$ which increases to $75.9 \%-100 \%$ when accompanied by severe stenosis or occlusion of the MCA or ICA. ${ }^{11,12}$ Maeda et $\mathrm{al}^{24}$ showed that the presence of FVH decreases from $100 \%$ within 24 hours of onset to $50 \%$ within 10-13 days of onset. In the present study, the proportion of severe stenosis or occlusion of the MCA was as high as $80.6 \%$, and most of these patients did not undergo MR imaging a short time after onset, which could explain the median prevalence of FVH (60.6\%). In addition, we saw no significant differences in the prevalence of FVH in our 3 groups, 


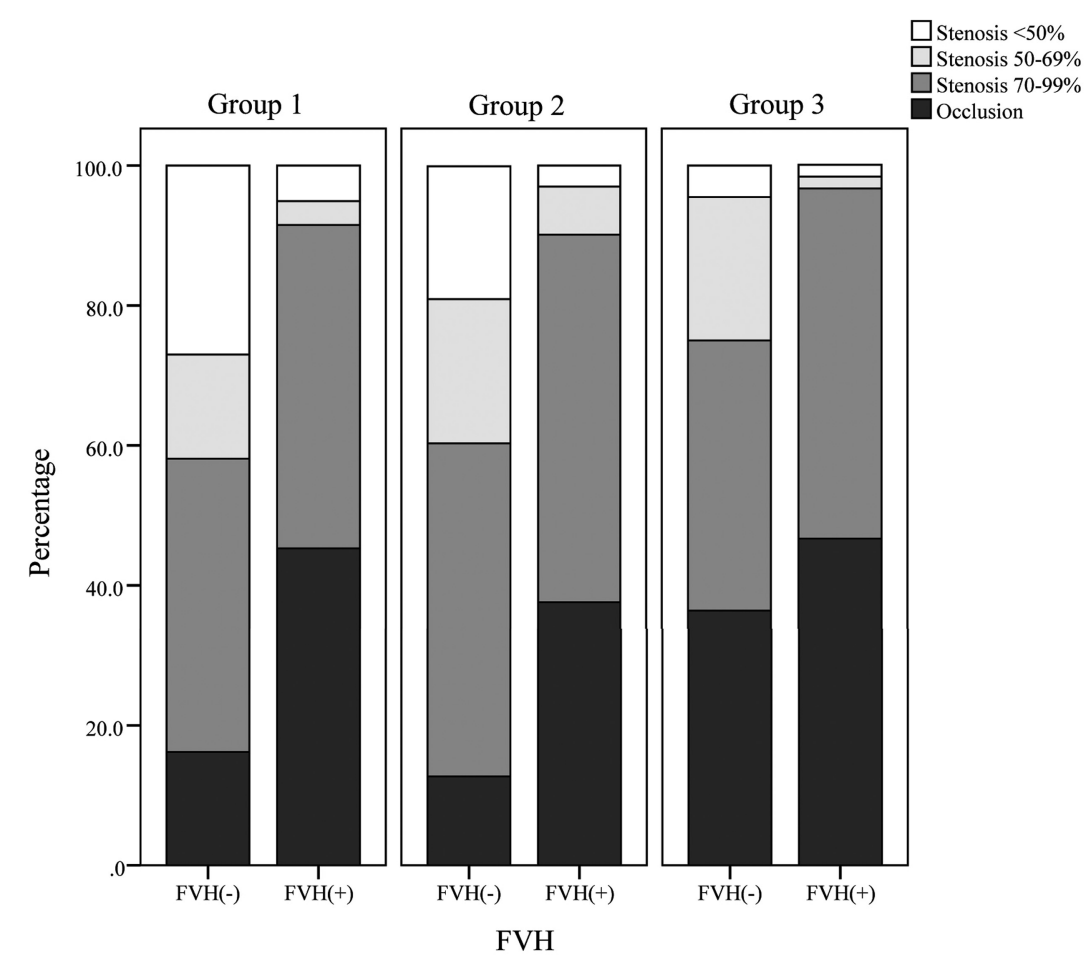

FIG 1. Detailed middle cerebral artery vascular status by symptom-to-MR imaging time, which increases with group number and the presence of FLAIR vascular hyperintensity.

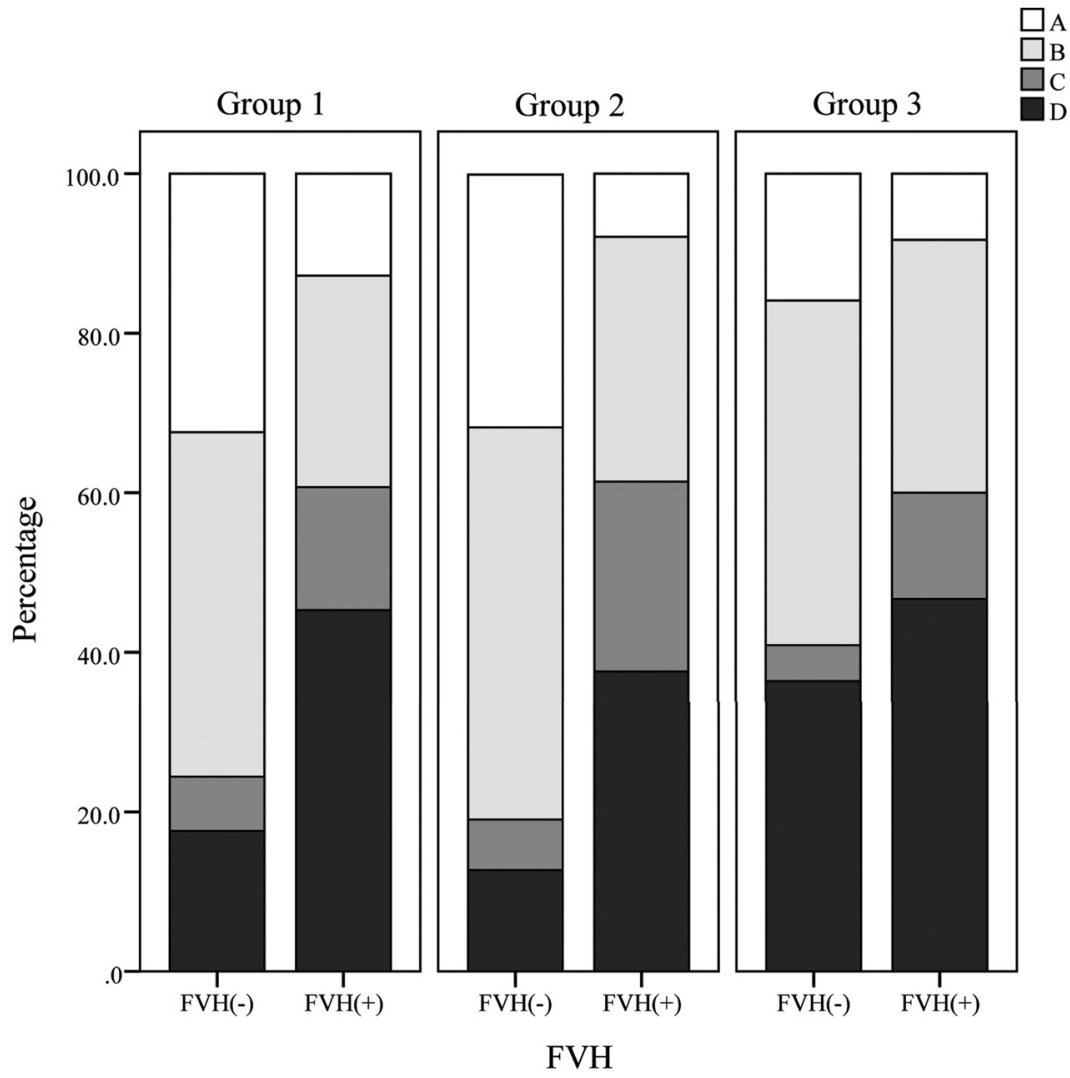

FIG 2. Detailed middle cerebral artery signal intensity by symptom-to-MR imaging time, which increases with group number and the presence of FLAIR vascular hyperintensity. A, All M3 branches of the MCA are visualized on the cortical surface. $B$, One or more $M 3$ branches are not visualized on the cortical surface. $C$, The superior or inferior trunk of the MCA or $\geq 1 \mathrm{M} 2$ branch is not visualized along its course. $D, M 1$ branches are not visualized along the course of the MCA. unlike Maeda et al. ${ }^{24}$ One reason could be that the proportion of severe stenosis or occlusion and the signal intensity of MCA were not significantly different in our 3 groups, and the Maeda et $\mathrm{al}^{24}$ study did not measure the degree of MCA lesion. Another possible reason is the much greater sample size in our study. The sample size in Maeda et $\mathrm{al}^{24}$ was 27 patients, with only 2 patients imaged within 10-13 days of onset, which would have led to serious errors.

The prognostic value of $\mathrm{FVH}$ has been widely investigated, ${ }^{4,6-23}$ with greatly divergent results. Besides populations, end points, and FVH classifications, we think that the symptomto-MR imaging time plays an important role in explaining the discrepancies in the literature. In our study, FVH (+) was associated with unfavorable clinical and radiographic outcomes within 6 hours to 14 days of onset, but these associations disappeared beyond 14 days of onset. On reviewing previous studies, we found that the symptom-to-MR imaging time was within 6 hours of onset or within the time window of reperfusion therapy in most of the studies that showed an association between FVH and good outcome. ${ }^{10,11,13-17}$ On the other hand, the symptom-to-MR imaging time was between 12 and 24 hours and several days in most of the studies showing an association between FVH and a poor outcome $e^{6-8,18-20}$; these results are similar to ours for the group within 6 hours to 14 days of onset.

We assumed that the role of FVH would vary with time. Within 6 hours of onset or within the time window of reperfusion therapy, FVH might be a marker of leptomeningeal anastomoses that are anatomically present and develop dramatically and rapidly after the onset of acute ischemic stroke. ${ }^{2,5}$ The areas with FVH, especially those with FVH-DWI mismatch, represent the brain tissue at risk of infarction, which could be saved by reperfusion therapy to reduce the final lesion volume and improve outcome. ${ }^{6,9,16,29}$ This may explain why patients with FVH have better outcomes than those without FVH in this period.

Beyond the time window of reperfusion therapy, however, the observation of persistent FVH may represent persis- 


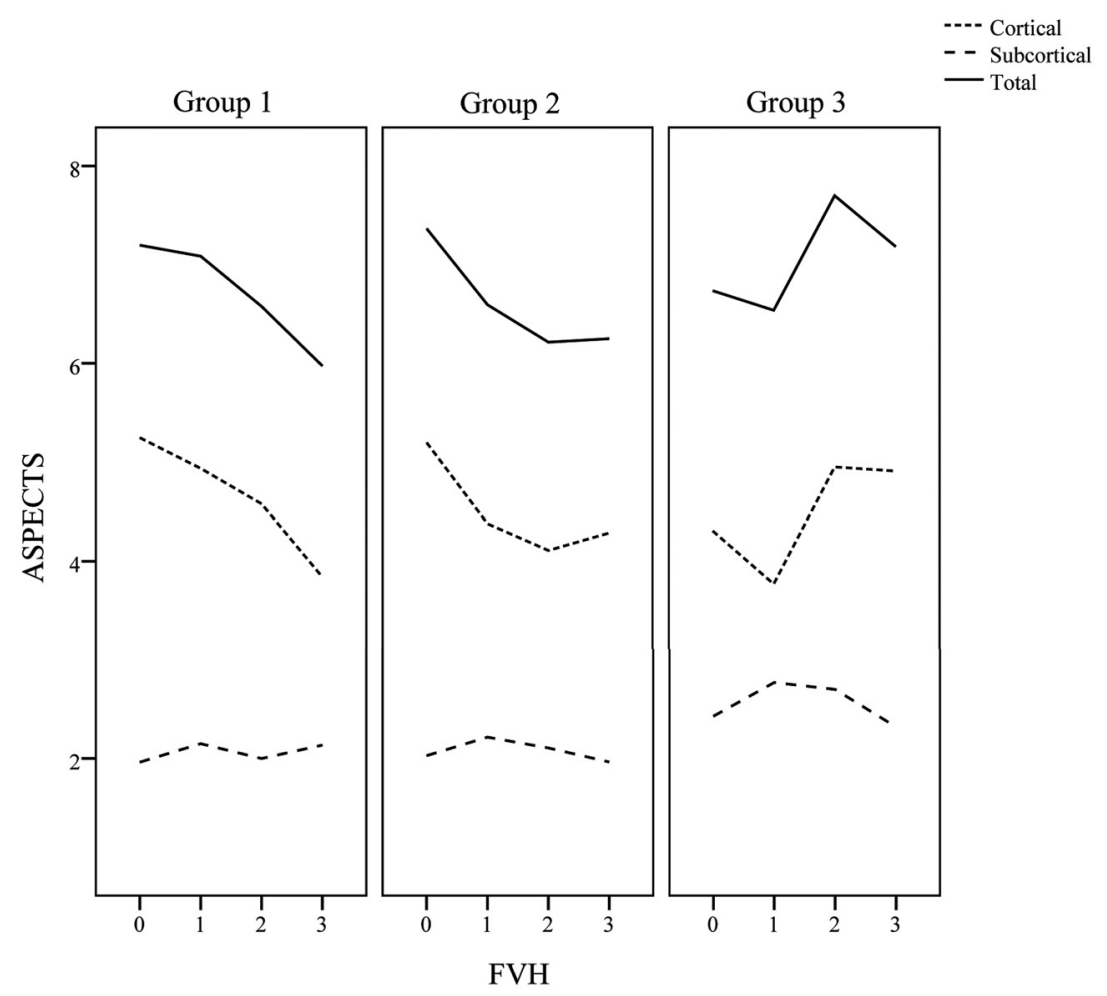

FIG 3. The ASPECTS lesion volume against the distal FLAIR vascular hyperintensity score.

our knowledge of the importance of the symptom-to-MR imaging time beyond 14 days.

Unlike most studies that focused on the superacute stage of ischemic stroke, we used a much longer time span after ischemic stroke. Consequently, our study conditions more closely resembled "real world" clinical scenarios. Patients can undergo MR imaging any time after stroke onset, and using only $\mathrm{FVH}$ to evaluate the superacute ischemic stroke stage is insufficient for clinical work. However, FVH is relatively easy to detect on MR imaging and may therefore reflect collateral status and potentially act as a substitute for conventional angiography. ${ }^{31}$

This study has some limitations. First, this is a retrospective clinical study. However, it shows, by analysis, a clear time dependency of the prognostic value of FVH. Further prospective studies are needed to confirm our findings. Second, we excluded patients with endovascular therapy. Although this ex-

tent vessel occlusion and impaired hemodynamics. ${ }^{4,14,29,30}$ Therefore, patients with FVH could be influenced much more by hemodynamic instability than patients without it. ${ }^{19}$ This difference might be correlated with a poor outcome in patients with FVH during this period. Some studies that re-examined MR imaging after the superacute period support this assumption. ${ }^{4,14,29}$ Sanossian et $\mathrm{al}^{14}$ found that a persistence of FVH on follow-up MR imaging was associated with a decreased or absent flow signal intensity on MRA, whereas the absence of FVH was seen in the setting of a return of the MR imaging flow signal intensity. This result is similar to our finding that patients with FVH had poorer MCA signal intensity. However, the observation of a decrease in FVH after reperfusion therapy predicted successful recanalization, smaller infarct volume, and good outcomes. ${ }^{4,29}$

Here, FVH was not significantly associated with clinical or radiologic outcome in group 3, though we continued to see a preponderance of patients with $\mathrm{FVH}$ who had severe stenosis or occlusion of the MCA. In addition, the infarct volume tended to decrease with increases in the distal FVH score in group 3, which is the opposite of the results in groups 1 and 2 . This outcome indicates that the wider distribution of distal FVH may be favorable when the symptom-to-MR imaging time extends beyond 14 days. We think that this possibility could be explained in the following 2 ways: First, new collaterals require time to develop significant capacity for cerebral blood flow. ${ }^{1,5}$ Second, achieving functional vessels through arteriogenesis requires several days to weeks. ${ }^{1,2}$ Therefore, these collaterals likely contribute more to alleviating the subacute or chronic stages of stroke than to mitigating acute ischemic events. The observation of no or little FVH in the subacute or chronic stages of stroke may represent an absence of effective collaterals. Future research should further refine clusion ensures data homogeneity and avoids the influence of a diversity of treatments on outcome, it leads to no patients receiving MR imaging within 6 hours of onset in this study. However, many studies have focused on the superacute stage of stroke, ${ }^{10,11,13-17,21,22}$ while few ${ }^{25}$ have focused on the subacute and chronic stages.

\section{CONCLUSIONS}

For patients with MCA infarctions, FVH $(+)$ is associated with unfavorable outcomes within 6 hours to 14 days of onset, while the wider distribution of distal FVH may be favorable beyond 14 days of onset. Therefore, the symptom-to-MR imaging time should be taken into account when assessing the prognostic value of FVH.

Disclosures: Wenjin Shang-RELATED: Grant: National Natural Science Foundation of China (No. 81671134); Medical Scientific Research Foundation of Guangdong Province of China (No. A2017219); Guangzhou Science and Technology Programme (No. 2014Y2-00502), Comments: My institution is supported by the grants from Guangdong Provincial Key Laboratory for Diagnosis and Treatment of Major Neurological Diseases (No. 2017B030314103); Southern China International Cooperation Base for Early Intervention and Functional Rehabilitation of Neurological Diseases (No. 2015B050501003); Guangdong Provincial Engineering Center for Major Neurological Disease Treatment; Guangdong Provincial Translational Medicine Innovation Platform for Diagnosis and Treatment of Major Neurological Disease. Liming M. ShuRELATED: Grant: National Natural Science Foundation of China (No. 81671134); Medical Scientific Research Foundation of Guangdong Province of China (No. A2017219); Guangzhou Science and Technology Programme (No. 2014Y2-00502).* Su XiaoRELATED: Grant: Guangdong Provincial Key Laboratory for Diagnosis and Treatment of Major Neurological Diseases (No. 2017B030314103); Southern China International Cooperation Base for Early Intervention and Functional Rehabilitation of Neurological Diseases (No. 2015B050501003); Guangdong Provincial Engineering Center for Major Neurological Disease Treatment; Guangdong Provincial Translational Medicine Innovation Platform for Diagnosis and Treatment of Major Neurological Disease.* Hua Hong—RELATED: Grant: National Natural Science Foundation of China (No. 81671134); Medical Scientific Research Foundation of Guangdong Province of 
China (No. A2017219); Guangzhou Science and Technology Programme (No. 2014Y200502), Comments: Our institution is supported by the grants from Guangdong Provincial Key Laboratory for Diagnosis and Treatment of Major Neurological Diseases (No. 2017B030314103); Southern China International Cooperation Base for Early Intervention and Functional Rehabilitation of Neurological Diseases (No. 2015B050501003); Guangdong Provincial Engineering Center for Major Neurological Disease Treatment; Guangdong Provincial Translational Medicine Innovation Platform for Diagnosis and Treatment of Major Neurological Disease; Others: We paid money to Editage, a division of Cactus Communications, for English editing to ensure that the language is clear and free of errors. The intent of our message was not altered in any way during the editing process. *Money paid to institution.

\section{REFERENCES}

1. Liebeskind DS. Collateral circulation. Stroke 2003;34:2279-84 CrossRef Medline

2. Iwasawa E, Ichijo M, Ishibashi S, et al. Acute development of collateral circulation and therapeutic prospects in ischemic stroke. $\mathrm{Neu}$ ral Regen Res 2016;11:368-71 CrossRef Medline

3. Bang OY, Goyal M, Liebeskind DS. Collateral circulation in ischemic stroke: assessment tools and therapeutic strategies. Stroke 2015;46:3302-09 CrossRef Medline

4. Ichijo M, Iwasawa E, Numasawa Y, et al. Significance of development and reversion of collaterals on MRI in early neurologic improvement and long-term functional outcome after intravenous thrombolysis for ischemic stroke. AJNR Am J Neuroradiol 2015;36: 1839-45 CrossRef Medline

5. Uemura A, O’Uchi T, Kikuchi Y, et al. Prominent laterality of the posterior cerebral artery at three-dimensional time-of-flight MR angiography in M1-segment middle cerebral artery occlusion. AJNR Am J Neuroradiol 2004;25:88-91 Medline

6. Kamran S, Bates V, Bakshi R, et al. Significance of hyperintense vessels on FLAIR MRI in acute stroke. Neurology 2000;55:265-69 CrossRef Medline

7. Kono T, Naka H, Nomura E, et al. The association between hyperintense vessel sign and final ischemic lesion differ in its location. $J$ Stroke Cerebrovasc Dis 2014;23:1337-43 CrossRef Medline

8. Cheng B, Ebinger M, Kufner A, et al; Stroke Imaging Repository (STIR) Investigators. Hyperintense vessels on acute stroke fluid-attenuated inversion recovery imaging: associations with clinical and other MRI findings. Stroke 2012;43:2957-61 CrossRef Medline

9. Toyoda K, Ida M, Fukuda K. Fluid-attenuated inversion recovery intraarterial signal: an early sign of hyperacute cerebral ischemia. AJNR Am J Neuroradiol 2001;22:1021-29 Medline

10. Mahdjoub E, Turc G, Legrand L, et al. Do fluid-attenuated inversion recovery vascular hyperintensities represent good collaterals before reperfusion therapy? AJNR Am J Neuroradiol 2018;39:77-83 CrossRef Medline

11. Liu D, Scalzo F, Rao NM, et al. Fluid-attenuated inversion recovery vascular hyperintensity topography: novel imaging marker for revascularization in middle cerebral artery occlusion. Stroke 2016;47: 2763-69 CrossRef Medline

12. Huang X, Liu W, Zhu W, et al. Distal hyperintense vessels on FLAIR: a prognostic indicator of acute ischemic stroke. Eur Neurol 2012;68: 214-20 CrossRef Medline

13. Olindo S, Chausson N, Joux J, et al. Fluid-attenuated inversion recovery vascular hyperintensity: an early predictor of clinical outcome in proximal middle cerebral artery occlusion. Arch Neurol 2012;69:1462-68 CrossRef Medline

14. Sanossian N, Saver JL, Alger JR, et al. Angiography reveals that fluidattenuated inversion recovery vascular hyperintensities are due to slow flow, not thrombus. AJNR Am J Neuroradiol 2009;30:564-68 CrossRef Medline

15. Lee KY, Latour LL, Luby M, et al. Distal hyperintense vessels on
FLAIR: an MRI marker for collateral circulation in acute stroke? Neurology 2009;72:1134-39 CrossRef Medline

16. Legrand L, Tisserand M, Turc G, et al. Do FLAIR vascular hyperintensities beyond the DWI lesion represent the ischemic penumbra? AJNR Am J Neuroradiol 2015;36:269-74 CrossRef Medline

17. de la Ossa N, Hernandez- Pérez M, Domènech S, et al. Hyperintensity of distal vessels on FLAIR is associated with slow progression of the infarction in acute ischemic stroke. Cerebrovasc Dis 2012;34: 376-84 CrossRef Medline

18. Nam KW, Kwon HM, Park SW, et al. Distal hyperintense vessel sign is associated with neurological deterioration in acute ischaemic stroke. Eur J Neurol 2017;24:617-23 CrossRef Medline

19. Kim SE, Lee BI, Kim SE, et al. Clinical significance of fluid-attenuated inversion recovery vascular hyperintensities in borderzone infarcts. Stroke 2016;47:1548-54 CrossRef Medline

20. Gawlitza M, Gragert J, Quäschling U, et al. FLAIR-hyperintense vessel sign, diffusion-perfusion mismatch and infarct growth in acute ischemic stroke without vascular recanalisation therapy. J Neuroradiol 2014;41:227-33 CrossRef Medline

21. Nave AH, Kufner A, Bücke P, et al. Hyperintense vessels, collateralization, and functional outcome in patients with stroke receiving endovascular treatment. Stroke 2018;49:675-81 CrossRef Medline

22. Kufner A, Galinovic I, Ambrosi V, et al. Hyperintense vessels on FLAIR: hemodynamic correlates and response to thrombolysis. AJNR Am J Neuroradiol 2015;36:1426-30 CrossRef Medline

23. Gawlitza M, Bohme J, Maros M, et al. FLAIR vascular hyperintensities and 4D MR angiograms for the estimation of collateral blood flow in anterior cerebral artery ischemia. PLoS One 2017;12: e0172570 CrossRef Medline

24. Maeda M, Koshimoto Y, Uematsu H, et al. Time course of arterial hyperintensity with fast fluid-attenuated inversion-recovery imaging in acute and subacute middle cerebral arterial infarction. J Magn Reson Imaging 2001;13:987-90 CrossRef Medline

25. Essig M, von Kummer R, Egelhof T, et al. Vascular MR contrast enhancement in cerebrovascular disease. AJNR Am J Neuroradiol 1996;17:887-94 Medline

26. Barber PA, Demchuk AM, Zhang J, et al. Validity and reliability of a quantitative computed tomography score in predicting outcome of hyperacute stroke before thrombolytic therapy: ASPECTS study group-Alberta Stroke Programme Early CT Score. Lancet 2000; 355:1670-74 CrossRef Medline

27. Lee DK, Kim JS, Kwon SU, et al. Lesion patterns and stroke mechanism in atherosclerotic middle cerebral artery disease: early diffusion-weighted imaging study. Stroke 2005;36:2583-88 CrossRef Medline

28. Hirooka R, Ogasawara K, Inoue T, et al. Simple assessment of cerebral hemodynamics using single-slab 3D time-of-flight MR angiography in patients with cervical internal carotid artery stenoocclusive diseases: comparison with quantitative perfusion singlephoton emission CT. AJNR Am J Neuroradiol 2009;30:559-63 CrossRef Medline

29. Sakuta K, Saji N, Aoki J, et al. Decrease of hyperintense vessels on fluid-attenuated inversion recovery predicts good outcome in t-PA patients. Cerebrovasc Dis 2016;41:211-18 CrossRef Medline

30. Liu W, Yin Q, Yao L, et al. Decreased hyperintense vessels on FLAIR images after endovascular recanalization of symptomatic internal carotid artery occlusion. Eur J Radiol 2012;81:1595-600 CrossRef Medline

31. Kim SJ, Ha YS, Ryoo S, et al. Sulcal effacement on fluid attenuation inversion recovery magnetic resonance imaging in hyperacute stroke: association with collateral flow and clinical outcomes. Stroke 2012;43:386-92 CrossRef Medline 